\title{
Improving Palmprint based Biometric System Performance using Novel Multispectral Image Fusion Scheme
}

\author{
Essia Thamri ${ }^{1}$, Kamel Aloui ${ }^{2}$, Mohamed Saber Naceur ${ }^{3}$ \\ LTSIRS Laboratory \\ National Engineering School Tunis \\ ENIT, Tunisia
}

\begin{abstract}
Nowadays, there are several identification systems which are based on different biometric modalities. In particular, multispectral images of palmprints captured in different spectral bands have a very distinctive biometric identifier. This paper proposes a novel fusion scheme of a biometric recognition system by multiSpectral palmprint. This system is composed of three blocks: (1) extraction of the region of interest (ROI) from multispectral images, (2) a new image fusion architecture based on the measurement of decorrelation, and (3) a scheme of dimension reduction and classification. The proposed image fusion system combines the information from the same left and right spectral band using the $2 \mathrm{D}$ discrete wavelet (DWT) transform technique. In addition, a feature extraction using the Log-Gabor transform is performed, while the feature size has been reduced using the Kernel Principal Component Analysis technique (KPCA). In Our experiments we use CASIA multispectral palmprint database. We obtained an accuracy rate (ACC) of $99.50 \%$ for the spectral bands WHT (white light) and $940 \mathrm{~nm}$ and an equal error rate $\mathrm{EER}=\mathbf{0 . 0 5 \%}$. These results show that our system is robust against spoofing.
\end{abstract}

Keywords-Biometric recognition; palmprint; multispectral images; image fusion; Log-Gabor; KPCA; DWT; CASIA

\section{INTRODUCTION}

The person biometric recognition is the measurement of certain biological parameters or physical character specific to each individual. [1, 2, 3, 4].A large number of various biometric modalities identification systems, such as, iris [5], fingerprint [6, 7] and palmprint [8] are unique and allow to identify person with certainty. Counteracting insecurity and external threat, several techniques have been developed. In this context, biometric recognition by multi-spectral palmprint has emerged as a very attractive alternative $[8,9,10,11$ ]. In addition, palmprints have the characteristics of stability, uniqueness and scalability, etc. [12]. This modality has a strong discrimination performance as well as an anti-noise capacity $[13,14]$. As a result, these biometric systems have predicted their reliability and robustness against spoofing. This may be due to the fact that this modality presents several information such as, ridges, main and fine lines [15]. Furthermore, vein features that are hidden can be easily detected with infrared light $[16,17,18]$. Also, to acquire these visible features, it is necessary to take the images of the palms with several spectral bands. Recently, the multispectral palmprint biometrics have captured attention over the past 15 decades [6, 19, 20, 21, 22].
The palmprint multispectral imaging system uses cameras to represent spectral bands, where each band is represented by a single camera that can acquire palmprint images on different bands [23, 24]. This imaging system offers more advantages because the recording task is not compulsory seen that all the different bands images are procured at the same time. The biometric system is very sensitive to the quality of acquisition $[25,26]$. Image acquisition can be done with or without contact. The system of recognition by the palmprint with contact is not acceptable by the users, since it is an environment of diseases transmission, therefore it is not recommended in the market. As well as the contactless biometric system is simple, comfort, less user contribution. So this system seems user-friendly and socially acceptable for person. As a result, it is highly recommended in the market. Despite the advantages of this non-contact biometric system, the acquired images contain several variations of scale between the images as well as the variation due to the translation and the rotation [27]. The multispectral palmprint algorithms focus on different multimodal fusion techniques: image-level fusion, at feature and at score-level. For image-level fusion, the goal is to obtain a single image by combining information from captured images for different spectral bands. For the scorelevel fusion technique, a feature extraction scheme of captured images at different spectral bands is used to have a comparison score that will be fused using a sum rule on which verification is performed. In literature, this rule has been frequently used, yet there is another alternative for this technique. Thus, the robustness of these multispectral biometric systems that use the image-level fusion approach generally depends on used fusion architecture. For the fusion technique, the use of feature extraction scheme is very interesting to obtain the performances of the biometric system. Several studies have shown that the fusion schemes of image-level are more efficient because these algorithms are robust to different types of noise due to lighting, motion blur, and so on [28,29]. However, the image fusion techniques have shown good extraction of details of the vein patterns and excellent reduction of noise in the image. Also, the technique of FPDCT and MRDCT could enhance the recognition rate [30]. In the work done by J.-G. Wang et al., a novel method of fusion by locality preservation projections (LPP) was developed [31]. This method improves the contrast, preserves the palm vein images and the edges. Also a new representation characteristic "Laplacian palm", which is extracted the features from the 
images fused by the technique of locality preservation projections (LPP) is developed [32]. The fusion score level showed good performance compared to that with a single band. Xingpeng et al. have presented a biometric identification system depending on Quarter Principle Component Analysis (QPCA) [33]. This method is presented on different fusion schemes: the Curvelet transform, the morphological pyramid, the wavelet transform, and the gradient pyramid. The results found show that the fusion based on the Curvelet transformation presents more qualitative information. Another feature extraction and image fusion scheme has been developed [34]. This method combines information from the different spectral bands. Using discriminant kernel analysis (KDA) a dimension reduction was performed before classification using a classifier (SRC). A new approach to recognition of palm prints has been presented in [35].This approach is based on AE (an optimized auto-encoder) (AE), RELM (regularized extreme learning machine) and an extraction method (HOG-SGF). The experiments were carried out on three databases (CASIA, MS-PolyU, and Tongji of contact less visible palmprint images). The HOG-SGF approach gives better accuracy results than the HOG descriptor. Therefore, in this paper, a new fusion scheme of a multispectral Palmprint recognition system is developed. First, a new method for decomposing palmprint images of the same right and left spectral band on several spectral bands is proposed. This fusion scheme can more precisely combine information from the same Right and Left spectral band according to the measure of dependence. To obtain a texture characteristic in different sub-bands the discrete wavelets transform 2D (DWT) is carried out in order to acquire this multi-scale decomposition. Works have been developed on the fusion of images of palm prints acquired in two spectral bands for example (460 nm and $940 \mathrm{~nm}$ ) [34]. On the other hand, our fusion method consists in applying 2D (DWT) on the same right and left spectral band. Our method shows excellent results compared to [34]. Secondly, a measure of the statistical dependence by the mutual information (MI) [36, 37] between the sub-bands has been developed. Finally, a fused palmprint image (left and right) is obtained by performing reverse DWT (IDWT) on fused wavelet coefficients. The accuracy rate
(ACC) of the proposed scheme was assessed using the multispectral CASIA database. In addition, experimental analysis of multispectral images of the palmprint has been performed to show the benefits of band fusion to enhance the recognition accuracy. The rest of the article is structured as follows: Section II presents the feature extraction method and the classification using three techniques: Transformation of Log-Gabor $[34,38]$ to effectively extract texture feature since our image is a texture image because there are repeating patterns, the Kernel Principal Component Analysis (KPCA) [40] to reduce dimensionality by minimizing redundancy [statistical dependence] and to evaluate the performance of our biometric system an Euclidean distance between the vectors is performed. Section III presents the different experimental results carried out with a comparison between works carried out and our proposed method. Finally, in section IV concludes this work.

\section{The Proposed Multispectral PALMPRint System}

Our developed system is composed of three steps: extraction of the region of interest (ROI), fusion of the (ROI) image followed by the extraction feature and the classification. Fig. 1 presents the proposed flowchart of our method of Multispectral palmprint fusion. Given the images of palm prints obtained are for different spectral bands. Doing the preprocessing of the images permit to extract the ROI firstly. Next, we present a novel multispectral image fusion scheme for the palmprint right and left of the same spectral band. So, that each multispectral image of the palmprint is broken down into two levels to get sub-bands using Haar wavelets. After that, we combine the sub-band information. To do that we use the mutual information MI to measure the dependence .Finally, we perform the feature extraction from the image fused by the Log-Gabor transform and classification to evaluate the system. By using the Log-Gabor we obtain a very high number of feature dimensions. Therefore, we carry out the reduction in dimensionality using the principal kernel component analysis technique (KPCA) before performing the classification using the Euclidean distance between the descriptors derived from (KPCA).

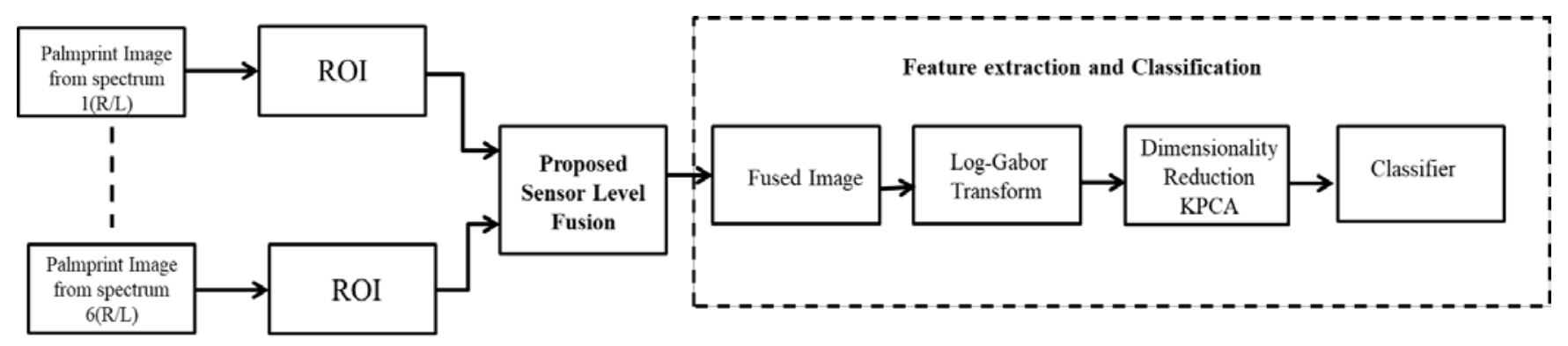

Fig. 1. Proposed Flowchart of our Method of Multispectral Palmprint Fusion. 


\section{A. Preprocessing and Palmprint Detection}

In this section we will develop the extraction of the region of interest (ROI) and the multi-spectral palmprint image fusion right and left of the same spectral band.

1) (ROI) extraction: The database used in this work is the multispectral palmprint CASIA. All the images of this database are acquired using a contactless sensor. So, there is a large rotational variation, scaling and translation [34, 40, 41, 42]. These images are taken in six spectral bands $700 \mathrm{~nm}, 460$ $\mathrm{nm}$, WHT(White Light), $630 \mathrm{~nm}, 850 \mathrm{~nm}$ and $940 \mathrm{~nm}[39,43]$. This database is very difficult for an accurate extraction of ROI. In this work, we used a ROI extraction scheme proposed by R. Rahavendra and C. Busch [34]. This scheme begins by carrying out the preprocessing phase of extract of the hand limit. The ROI is extracted with accuracy by detecting the benchmarks of the hand. Rahavendra and Busch detected two different landmarks R1 and R2 (see Fig. 2). To identify the binary discontinuities the scanning of the preprocessed image is involved for landmark detection. The choice of this method is based on its robustness since it allows, for different hand position, to estimate the translation and rotation corrections which are extremely hard to get when using contactless palmprint samples. Also this method gives a higher accuracy as indicated in Table I which compares the performance of the

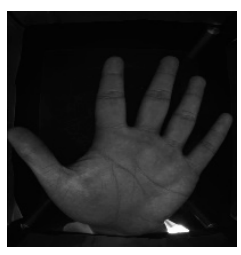

001_1_460_01

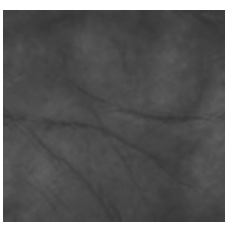

001_l_460_01

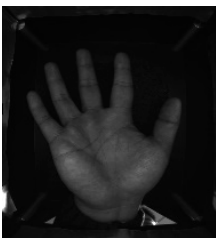

001_r_460_01

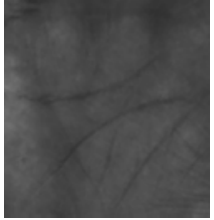

001_r_460_01

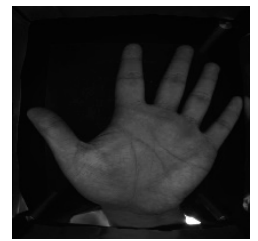

001_1_460_02

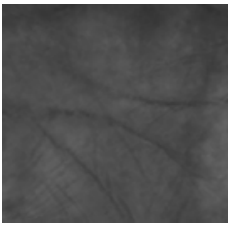

001_1_460_02

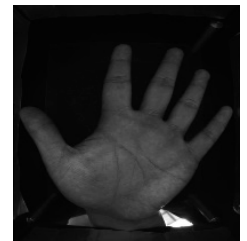

001_1_460_03

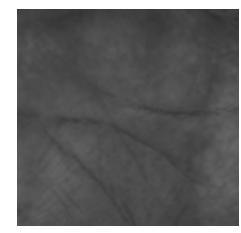

001_1_460_03
ROI extraction for the two methods used by Rahavendra and Bush [34], and khan et al [44].

Fig. 3 and 4 show the ROI extraction results at $460 \mathrm{~nm}$ (hand images Right and Left).

TABLE I. PERFORMANCE OF THE ROI EXTRACTION SCHEME OF RAHAVENDRA AND BUSH

\begin{tabular}{|l|l|}
\hline Methods & Accuracy (\%) \\
\hline Rahavendra and Busch [34] & 95 \\
\hline Khan et al. [44] & 90 \\
\hline
\end{tabular}

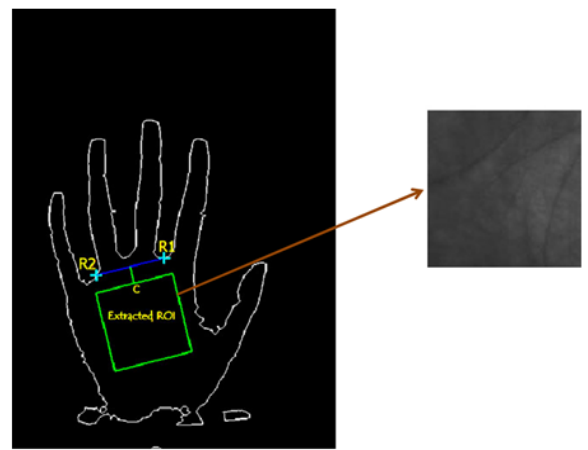

Fig. 2. ROI Extraction of Rahavendra and Busch.

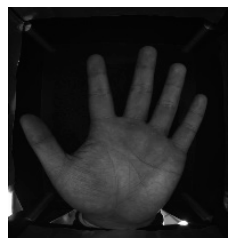

001_1_460_04

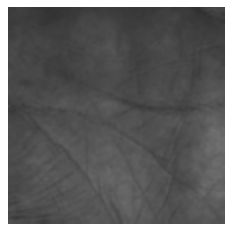

001_1_460_04

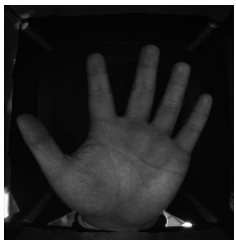

001_l_460_05

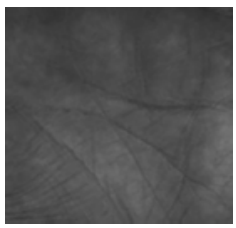

001_1_460_05

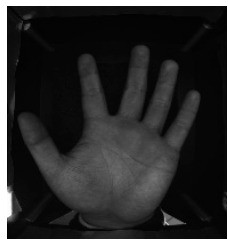

001_l_460_06

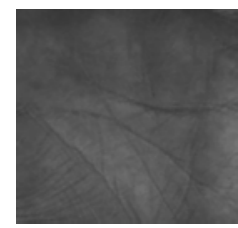

001_1_460_06
Fig. 3. ROI Extraction Results at $460 \mathrm{~nm}$ (Hand Images Left).

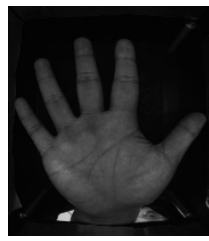

001_r_460_02

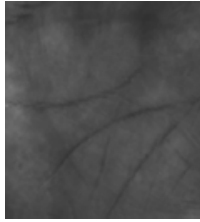

001_r_460_02

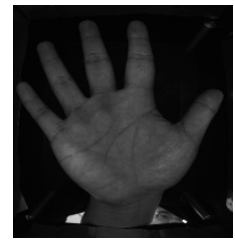

001_r_460_03

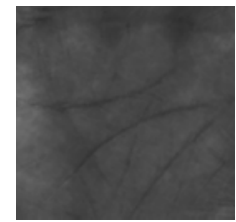

001_r_460_03

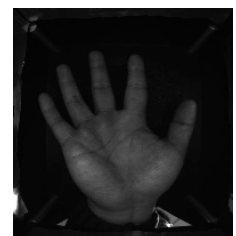

001_r_460_04

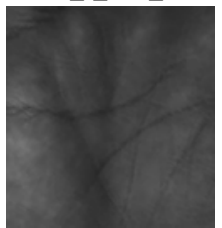

001_r_460_04

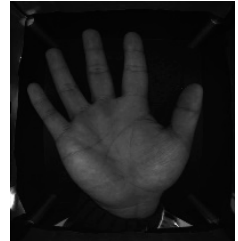

001_r_460_05

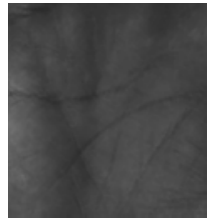

001_r_460_05
Fig. 4. ROI Extraction Results at $460 \mathrm{~nm}$ (Hand Images Right).

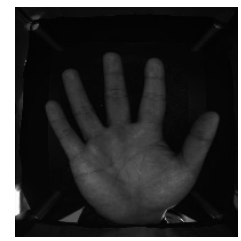

001_r_460_06

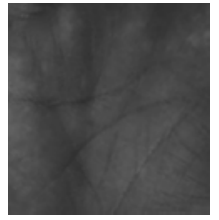

001_r_460_06 
2) The proposed fusion scheme: The proposed scheme for multi-spectral palmprint image fusion right and left of the same spectral band is described in this section.This approach is based on three steps. Firstly, using 2D (DWT) with Haar mother wavelet we carry out the multi-scale decomposition of the palmprint images. The decomposition can be carried out by several techniques such as wavelet transformation [45], Laplacian pyramid [43], etc. We have chosen the discrete wavelet transform 2D (DWT) because it is easy to implement and it includes less computational requirements [33, 45, 46, 47]. As a result of this approach we find the wavelet coefficients at sub-bands. Secondly, these sub-bands obtained from palm-print images are combined. Thirdly, to get a fused palmprint left and right the inverse DWT (IDWT) image is performed. By considering the example of images get at two spectral bands right and left of the $460 \mathrm{~nm}$ band, we will explain the proposed image fusion and will show this approach can be extended for $\mathrm{N}$ different bands. The wavelet coefficients shown in Fig. 6 and which are represented in seven different sub-bands constitutes the result of palmprint image decomposition from the first spectral band using the 2level DWT. From the first to the sixth sub-bands represent the approximation coefficients ( $\left.A_{1}\right)$, the horizontal detail coefficients $\left(\mathrm{H}_{1}\right.$ and $\left.\mathrm{H}_{2}\right)$, the vertical detail coefficients $\left(\mathrm{V}_{1}\right.$ and $\mathrm{V}_{2}$ ) and the diagonal coefficients $\left(\mathrm{D}_{1}\right.$ and $\mathrm{D}_{2}$ ) respectively.We apply the same procedure to the palmprint image from the Right band. As a result seven subbands: $\mathrm{H}_{1}^{\prime}, \mathrm{A}_{1}^{\prime}, \mathrm{H}_{2}^{\prime}, \mathrm{V}_{1}^{\prime}, \mathrm{V}_{2}^{\prime}, \mathrm{D}_{1}^{\prime}$ and $\mathrm{D}_{2}^{\prime}$. The degree of dependency between these sub-bands from the Left spectral band and the right spectral band (for example $A_{1}$ and $A_{1}^{\prime}$ ) is determined by utilising mutual information in the next step [36]. To quantify the dependence we have taken into account its ability to treat the non-Gaussian distribution and its robustness to the reduced size of the set of observations using mutual information. The mutual information between $A_{1}$ and $\mathrm{A}_{1}^{\prime}$, are defined as follows:
$M_{1}\left(A_{1}, A_{1}^{\prime}\right)=\left[\log \left(\frac{f\left(A_{1}, A_{1}^{\prime}\right)}{f\left(A_{1}\right) f\left(A_{1}^{\prime}\right)}\right)\right]$

With $\mathrm{A}_{1} \in S_{1}$ and $\mathrm{A}_{1}^{\prime}, \in S_{2}$ the two sub-bands where $S_{1}$ represents the image of the left spectral band and $S_{2}$ represents the image of the right spectral band. $f\left(\mathrm{~A}_{1}, \mathrm{~A}_{1}^{\prime}\right)$ represents a joint distribution. In equation (1), the mutual information $M_{1}\left(\mathrm{~A}_{1}, \mathrm{~A}_{1}^{\prime}\right)$ indicates how much the information $\mathrm{A}_{1}$ is transmitted to $\mathrm{A}_{1}^{\prime}$. The value of $M_{1}\left(\mathrm{~A}_{1}, \mathrm{~A}_{1}^{\prime}\right)=0$ shows that $\mathrm{A}_{1}$ and $A_{1}^{\prime}$ do not represent any common information. Similarly, when $M_{1}\left(\mathrm{~A}_{1}, \mathrm{~A}_{1}^{\prime}\right)$ has the highest value, this shows that the two sub-bands are dependent. We have estimated the density functions by the Parzen window with a Gaussian kernel [13].With this approach, we estimate well the distribution of sub-band wavelet coefficients [48].Using Eq. (1) we normalize mutual information values between 0 and 1 with sigmoidal normalization [49]. Then, a threshold (th) is compared with this value. When $M_{1}\left(\mathrm{~A}_{1}, \mathrm{~A}_{1}^{\prime}\right)$ value is greater than the threshold so it shows that $A_{1}$ and $A_{1}^{\prime}$ are linked to each other, so they must necessarily have the common information. Therefore, to perform the fusion we make the selection between $A_{1}^{\prime}$ and $A_{1}$ and. Then we choose between these sub-bands $A_{1}$ and $A_{1}^{\prime}$ which should be selected. Next, we calculate the energy of the wavelet coefficients in the sub-bands $A_{1}^{\prime}$ and $A_{1}$ distinctly. The one who has the highest energy is then selected. Instead, if the value $M_{1}\left(\mathrm{~A}_{1}, \mathrm{~A}_{1}^{\prime}\right)$ is equal or less than the threshold, then fusion between $A_{1}$ and $A_{1}^{\prime}$ is done, since the two sub-bands have complementary information. We represent the functional relation between the threshold and the mutual information as follows:

$$
\begin{aligned}
& w_{1}=\left(\frac{1-M_{1}\left(A_{1}, A_{1}^{\prime}\right)}{1+t h}\right) \\
& w_{2}=1-w_{1}
\end{aligned}
$$

The higher energy sub-band has the largest weights. We repeat this procedure on the others sub-bands. Then the (IDWT) is performed to obtain the fusion of multispectral palmprint image. Fig. 5 shows the proposed fusion scheme for six spectral bands. We extended this scheme for $\mathrm{N}$ bands.

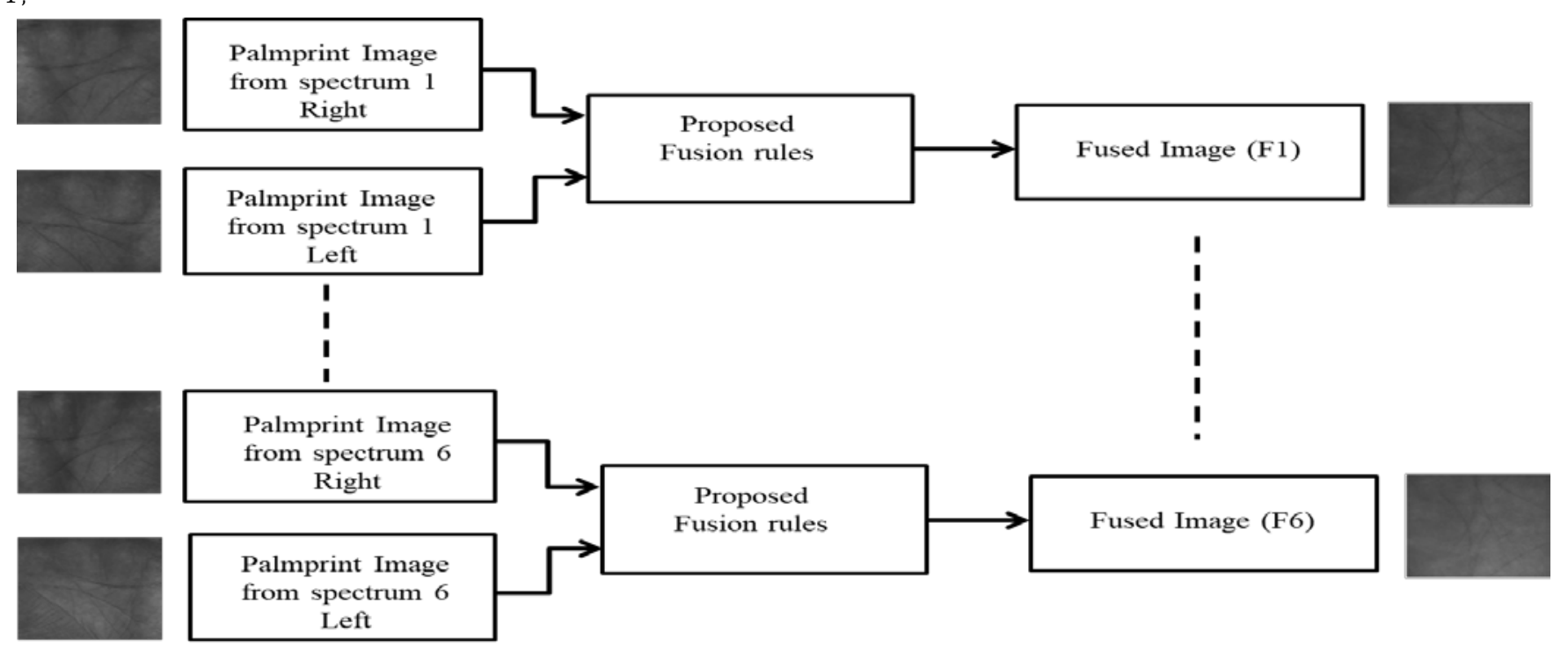

Fig. 5. Illustration of the Fusion Scheme for the 6 Bands. 


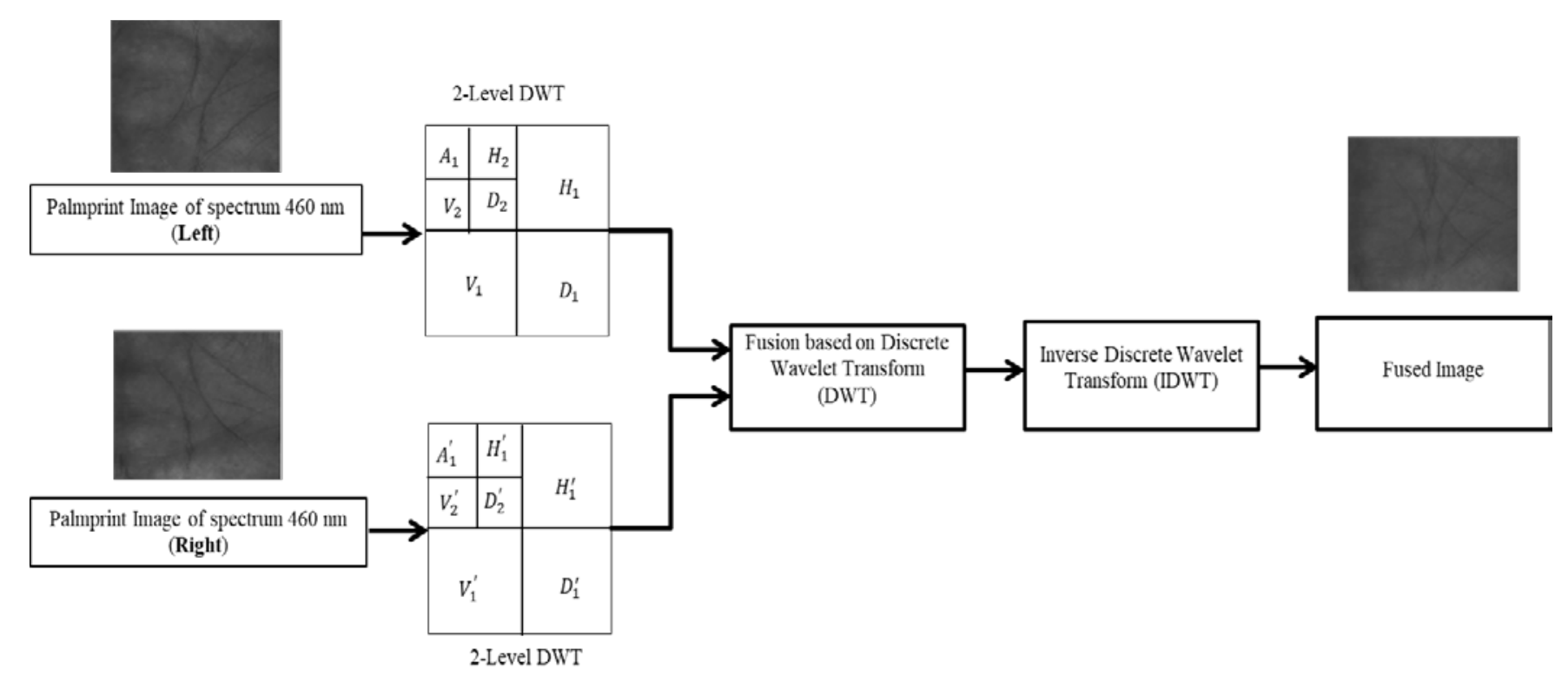

Fig. 6. Illustration of Fused Palmprint Image on Two Spectral Left and Right (460nm) Bands.

\section{B. Feature Extraction and Classification}

We get to the main line feature extraction step which is performed using the Log Gabor filter since the fused palmprint image is a texture image. The Log Gabor filter shows an improvement in performance in terms of precision [23] compared to the Gabor filter. In experiments The Log Gabor transform has eight orientations and four different scales. So, we have fixed these values after tests and in accordance with the literature [12]. Considering $X_{f p}$, the fused palmprint image whose size is $200 \times 200$. When we perform the Log Gabor transform $X_{f p}$ we get 32 images. The $X_{L G f p}$ image is the resulting Log Gabor transform image whose size is $420 \times 840$ [12]. We then performed a subsampling of the $X_{L G f p}$ image in a ratio equal to 6 to reduce the computation load, and we obtained an important compromise among the computation reduction and the degradation of the performance. Thus, the size of $X_{L G f p}$ is now reduced to $70 \times 140$. By concatenating the $X_{L G f p}$ columns we obtain a $X_{L G f p}$ descriptor vector with a size of $20,480 \times 1$. This procedure is repeated in all the remaining samples.

$X_{\text {GRef }}=\left(20,480, N_{\text {Ref }}\right)$ and $X_{G T e s}=\left(20,480, N_{\text {Tes }}\right)$

$N_{R e f}$ and $N_{T e s}$ are the numbers of reference and probes samples. Then we went to the reduction of dimensions of descriptor vectors to minimize redundancy before performing the classification. The Log Gabor transform each fused image gave us a high dimension of descriptor vector. However, for dimension reduction there are two types of data, non-linear and linear. The linear type has a difficult disadvantage of visually representing the object data in a graph and especially when using big data. In this work we used Kernel Principal Component Analysis (KPCA) as a reduction technique, which is a non-linear projection method and it is successfully used in biometrics [50, 51, 52]. This approach has proven its great capacity for reducing space and its good discriminating quality compared to the other approach. According to Kusban [54, 55], a kernel can be founded from 'a multiplication matrix with its transposition which can be presented as follows:

$k\left(x_{i}, x_{j}\right)=\Phi\left(x_{i}\right)^{T} \Phi\left(x_{j}\right)$

With $\left(x_{i}, x_{j}\right)$ : For the KPCA process

\section{$T$ : For transpose operation}

$\Phi\left(x_{i}\right)$ has a very large value so we have to midpoint of centered features $\widehat{\Phi}\left(x_{i}\right)$. Therefore, equation (3) can be modified as follows:

$k\left(x_{i}, x_{j}\right)=\widehat{\Phi}\left(x_{i}\right)^{T} \widehat{\Phi}\left(x_{j}\right)$

The equation (4) can be written as :

$k_{c}=k-2 l k+l k l$

With $l$ is $[\mathrm{N} \times \mathrm{N}]$ matrix with the value of $1 / \mathrm{N}$ for all elements. During our research on the novelty of the researcher authors, we found that the midpoint determination in the equation (4) is a critical value in KPCA [53]. So, we added several point values which can be presented as follows:

$k\left(x_{i}, x_{j}\right)=\left[\widehat{\Phi}\left(x_{i}\right)^{T} \widehat{\Phi}\left(x_{j}\right)\right]^{\sqrt{2}}$

We can also represent the new form of central nucleus in equation (5) as follows:

$k_{c}=k-l k-k l+l k l$

The $k$ amount data are reduced without losing significant features. The dimension reduction process must be carried out with the diagonal value of Singular Value Decomposition (SVD). To properly characterize the data in the discriminant sub-space of the kernel, the efficiency of the KPCA is related to type of used kernel function. So, in this article, we used the polynomial kernel of degree 2 to characterize the Log-Gabor features. Therefore, the use of KPCA permit to reduce the dimension of $X_{G R e f}$ and $X_{G T e s}$ by $X_{k p c a_{R e f}}=\left(838, N_{R e f}\right)$ and $X k p c a_{T e s}=\left(838, N_{T e s}\right)$. 


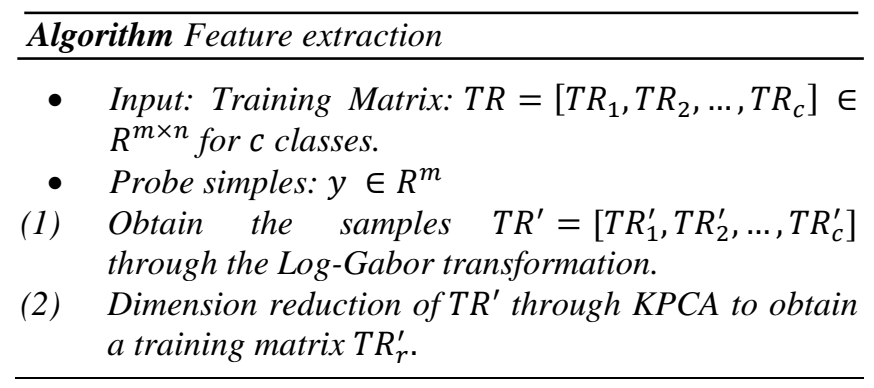

Once the step of the dimension reduction is finished, we passed to the step of the performance evaluation on by applying to Euclidean Distance-Based Matching between the vectors resulting from (KPCA).In our work we used $2 / 3$ of data for the train and $1 / 3$ for the test. In general, to evaluate the efficiency of the biometric system we are interested in two metrics of the performance variation FAR and FRR.

\section{EXPERIMENTAL RESULTS AND DISCUSSION}

We describe in this section the implemented protocol, the development environment, the data base representation and the results of our fusion method. The hardware and software tools used for the implementation of our solution are a personnel computer. Processor: Intel Core i5, CPU 2.4 GHz, Memory: 4 GB and Operating system: Windows 10 (32-bit). Also we use Matlab R2016b as simulation software.

\section{A. Database Description}

In this article, the database used is CASIA multispectral palmprint [43]. This database contains 200 hand images (100 right and 100 left). Each hand image has 6 samples and each sample contains six spectral bands $(700 \mathrm{~nm}, 630 \mathrm{~nm}, 460$ $\mathrm{nm}, 940 \mathrm{~nm}, 850 \mathrm{~nm}$ and WHT (white light)), which give a total of 7200 multispectral palmprint images.

\section{B. Motivation and Contributions}

The first phase of experiments is the preprocessing phase which consists in detecting the regions of ROI interest of palmprints images from our CASIA -Multispectral database. Then, we performed a fusion at the descriptor level of the right and left image of the same spectral band of the six bands (700 nm, $630 \mathrm{~nm}, 460 \mathrm{~nm}, 940 \mathrm{~nm}, 850 \mathrm{~nm}$ and WHT (white light)) by $2 \mathrm{D}$ discrete wavelet transform technique (DWT). The results of our fusion system showed excellent results compared to [34]. These results are shown in Table II. Then, we have to go to the main line features extraction phase using the LogGabor filter which allows to highlight the textures. Our image is an image of texture since it has repeating patterns. The next phase is the descriptor vector dimension reduction phase to minimize redundancy. The approach used for the reduction of dimension is the Kernel Principal Component Analysis technique (KPCA). To evaluate the performance of our biometric system, we applied the Euclidean-distance matching (EuM) technique between the vectors from KPCA.

\section{Comparison and Discussion}

In our research we have studied the recent methods of multispectral recognition of the palm print proposed in recent years. We have presented in Table II the different methods with their best performance rates for the two phases (verification and / or identification). We have analyzed and compared these rates. The methods studied are the methods of [34], [54], [55], [56] which use the multispectral palmprint CASIA database. The best performance results of these methods are found $95 \%$ in terms of Classification Accuracy (ACC) for identification and between [0.02; 3.12] \% in terms of EER for the verification mode. The approach proposed in [34] allow to fused images for a multispectral palmprint system by combining information coming from different spectral bands using Kernel Discriminant analysis (KDA). This approach is based on log-Gabor filters for features detection. The classification is done using a sparse representation classifier (SRC). This method allows obtaining an EER rate of $1.64 \%$ (fusion of all bands). In other works, the method proposed in [55] shows a new modified radon transformation method as well as the kernel principal component analysis is performed. The experiments used two databases Poly $U$ and CASIA palm print. The EER rate obtained is $0.02 \%$ in the CASIA database. Moreover, some methods [57] proposed a palmprint authentication system using Gabor wavelets for extraction coefficients, wavelet decomposition for palm image fusion, and colony optimization system. They use SVM to classify the samples. This approach permit to obtain an EERs of $3.97 \%$ and $3.12 \%$ on the evaluation sets. Moreover, a bidirectional matching technique has been used in place of a unidirectional matching in [54]. The algorithm used is founded on the strategy a feature-level fusion and sampling. EER rates for this method are $0.16 \%$ and $0.73 \%$ for two databases. In [56], the method seeks to represent a biometric identification system utilizing PCANet ${ }^{S_{2}}$ deep learning. This method is done by the 4 classifiers (RFT, SVM, KNN and RBF). The best ERR rate produced by the spectral band $(460 \mathrm{~nm})$ is $0.12 \%$.

Evaluating the performance of our system constitute the goal of the last part of this work. After the fusion of Left and Right spectral bands of the same band, a reduction of dimension is carried out by the KPCA. Then, for all the spectral bands, a Euclidean distance between the vectors issuing from KPCA is applied with the best parameters. Table III presents the results for each spectral band, permitting to see the performances of our proposed system. This table shows that the EER reaches the highest value ERR $=0.05 \%$ for to the two spectral bands $940 \mathrm{~nm}$ and WHT. Also, EER $=1.00 \%$ is achieved by the spectral bands $630 \mathrm{~nm}, 850 \mathrm{~nm}, 460 \mathrm{~nm}$ and an EER $=1.50 \%$ for the $700 \mathrm{~nm}$ spectral bands. The efficiency of all the spectral bands is illustrated in Fig. 9. This figure shows the Receiver Operating Characteristic (ROC) curves for the different spectral bands .A biometric system is said to be efficient if it has a small EER value. In this table, the best accuracy rate (ACC) in the case of two spectral bands $940 \mathrm{~nm}$ and $\mathrm{WHT}=99.50 \%$. For the $850 \mathrm{~nm}, 460 \mathrm{~nm}$ and $630 \mathrm{~nm}$ spectral bands the ACC $=99.00 \%$ and the $700 \mathrm{~nm}$ band at ACC $=98.50 \%$. Therefore the results show that our Biometric system has reached performance and approached the robustness part comparable to the best of advanced systems like those of [34.56]. Finally, in order to obtain the performance evaluation, an Euclidean distance is applied to each band between the vectors resulting from KPCA. Then the performance of the identification system is evaluated using classifier information. 
Table IV shows the accuracy variation for different threshold values (th) for the $940 \mathrm{~nm}$ (Right and Left) spectral band. The value of (th) varies from 0 to 1 . Fig. 7 shows the illustrating of the Accuracy of the spectral band 940nm for different (th) threshold values. We note that the best Accuracy value is obtained for the optimal decision threshold (0.5). Fig. 8 presents the proposed fusion scheme for six spectral bands. We extend this scheme for $\mathrm{N}$ bands.
Taking into account these accuracies, we think that our fusion approach to the level of the left and right image of the same spectral band (technique (DWT) + Kernel PCA) can be sufficient to represent the majority of biometric features within the biometric modality.

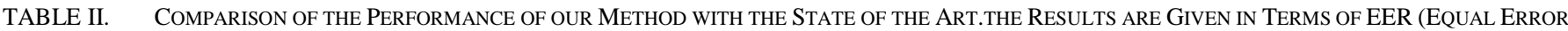
RATE) AND CLASSIFICATION ACCURACY (ACC)

\begin{tabular}{|c|c|c|c|}
\hline Method & Feature extraction & Database & Best recognition performance \\
\hline Raghavendra et al. (2014) [34] & Log-Gabor filter+KDA & CASIA & $\begin{array}{l}\text { Verification: EER }=1.640 \%(\text { fusion of all bands }) \\
\text { Identification: ACC }=95 \%\end{array}$ \\
\hline Kisku et al. (2012) [55] & Gabor wavelet transform & CASIA & $\begin{array}{l}\text { Verification: EER = } 0.0248 \% \\
\text { Identification: / }\end{array}$ \\
\hline Kisku et al. (2010) [57] & Wavelet Fusion with ACO & CASIA & $\begin{array}{l}\text { Verification: EER = 3.125\% } \\
\text { Identification: / }\end{array}$ \\
\hline Yan et al. (2015) [54] & RootSIFTbased feature fusion & CASIA & $\begin{array}{l}\text { Verification: EER }=0.160 \% \\
\text { Identification: / }\end{array}$ \\
\hline Meraoumia et al. (2017) [56] & Two-stage PCANet ${ }^{S_{2}}$ & CASIA & $\begin{array}{l}\text { Verification: EER=0.125\%(best 640nm) } \\
\text { Identification: / }\end{array}$ \\
\hline Proposed Method & Log-Gabor filter+KPCA & CASIA & $\begin{array}{l}\text { Verification: EER }=0.05 \% \text { (best } 940 \mathrm{~nm} \text { and WHT) } \\
\text { Identification: } 99.5 \% \text { (best } 940 \mathrm{~nm} \text { and WHT) }\end{array}$ \\
\hline
\end{tabular}

TABLE III. IDENTIFICATION SyStem PERFormance OF FuSED BANDS (CASIA DATABASE)

\begin{tabular}{|l|l|l|}
\hline Spectral fused Bands(nm) & EER (\%) & Accuracy (\%) \\
\hline 630 & 1.00 & 99.00 \\
\hline 700 & 1.50 & 98.50 \\
\hline 850 & 1.00 & 99.00 \\
\hline 460 & 1.00 & 99.00 \\
\hline 940 & 0.05 & 99.50 \\
\hline WHT & 0.05 & 99.50 \\
\hline
\end{tabular}

TABLE IV. ACCURACY VARIATION FOR DIFFERENT THRESHOLD VALUES (TH) FOR THE 940 NM (RIGHT AND LEFT) SPECTRAL BAND

\begin{tabular}{|l|l|}
\hline \multirow{2}{*}{ Threshold (th) values } & Accuracy (\%) \\
\cline { 2 - 3 } & Proposed Fusion scheme \\
\hline 0 & 99.30 \\
\hline 0.1 & 99.19 \\
\hline 0.2 & 99.29 \\
\hline 0.3 & 99.17 \\
\hline 0.4 & 99.49 \\
\hline $\mathbf{0 . 5}$ & $\mathbf{9 9 . 5 0}$ \\
\hline 0.6 & 99.41 \\
\hline 0.7 & 99.21 \\
\hline 0.8 & 99.05 \\
\hline 0.9 & 99.04 \\
\hline 1 & 99.21 \\
\hline
\end{tabular}




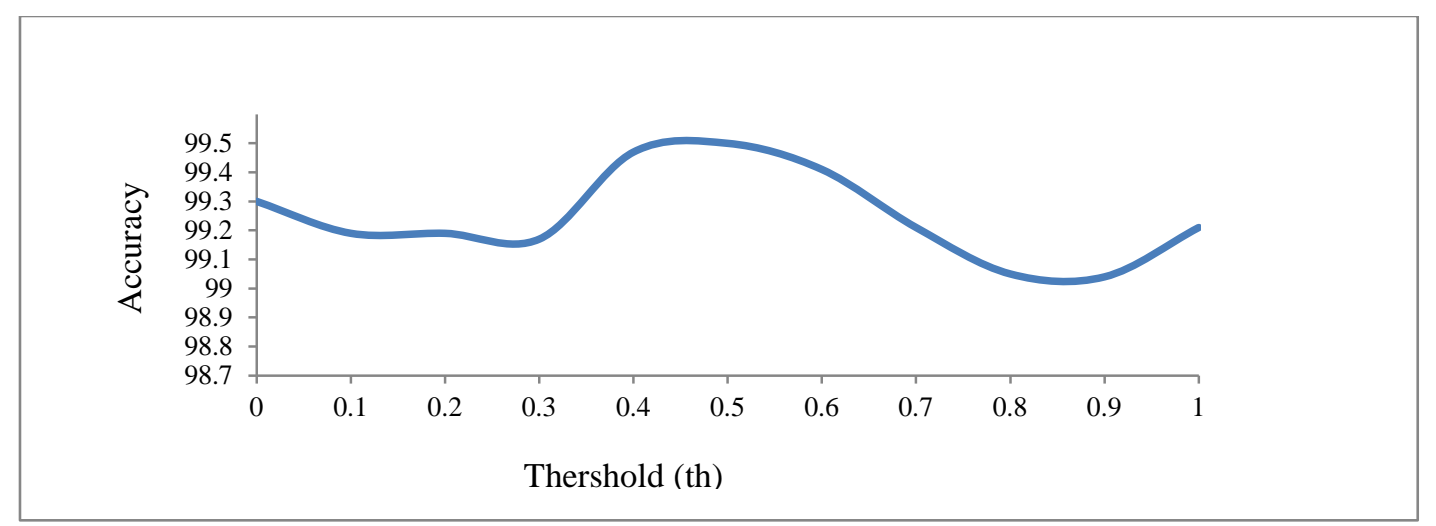

Fig. 7. Illustrating of the Accuracy of the Spectral Band 940nm (Right and Left) for different (th) threshold Values.

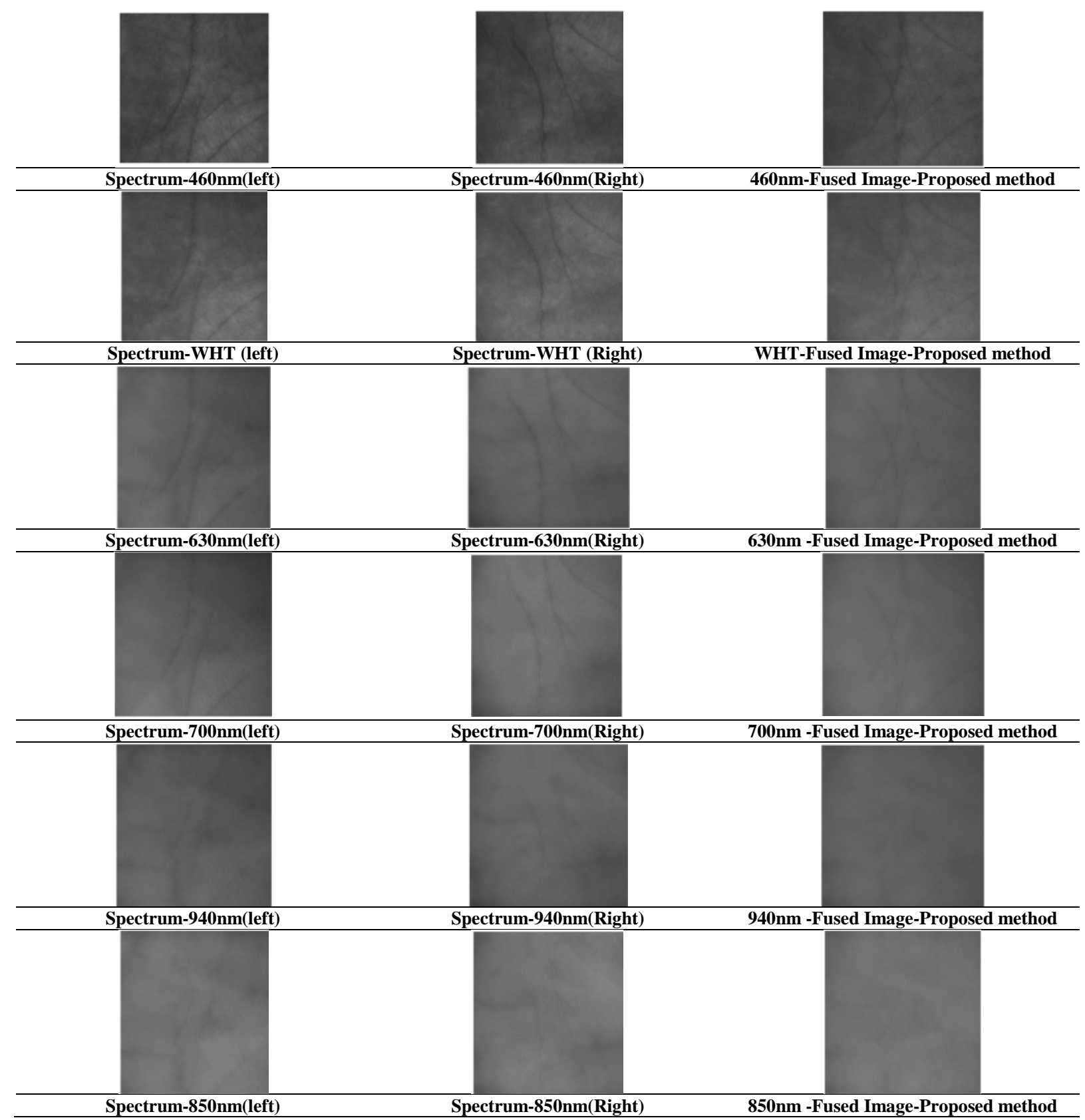

Fig. 8. Results of the Image Fusion Scheme Proposed on the Left and Right Spectral Bands of 6 Bands (700 nm, 460 nm, WHT, 630 nm, 850 nm and 940 nm). 


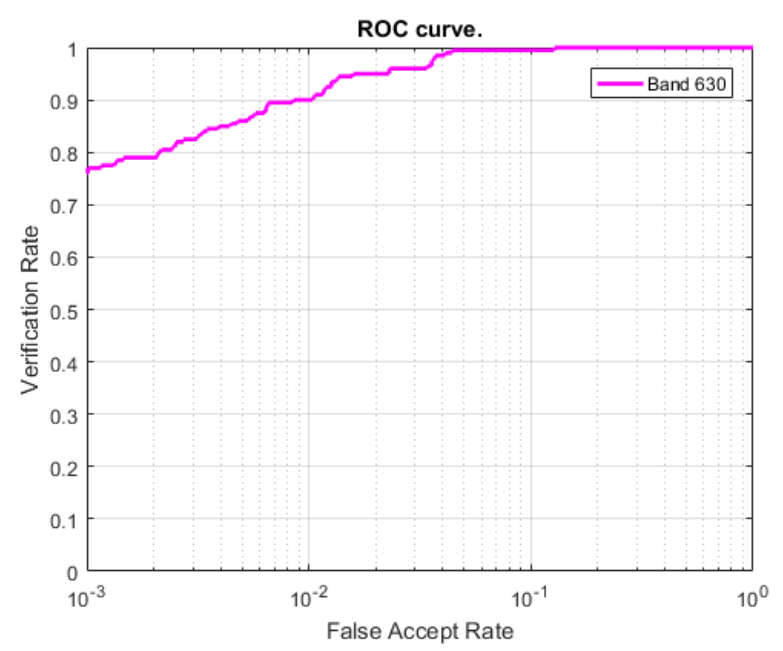

(a)

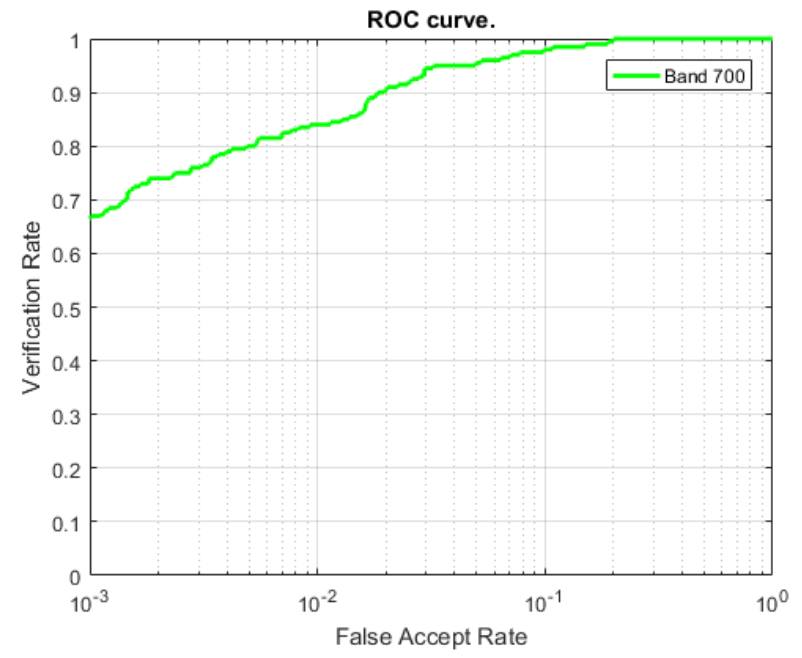

(c)

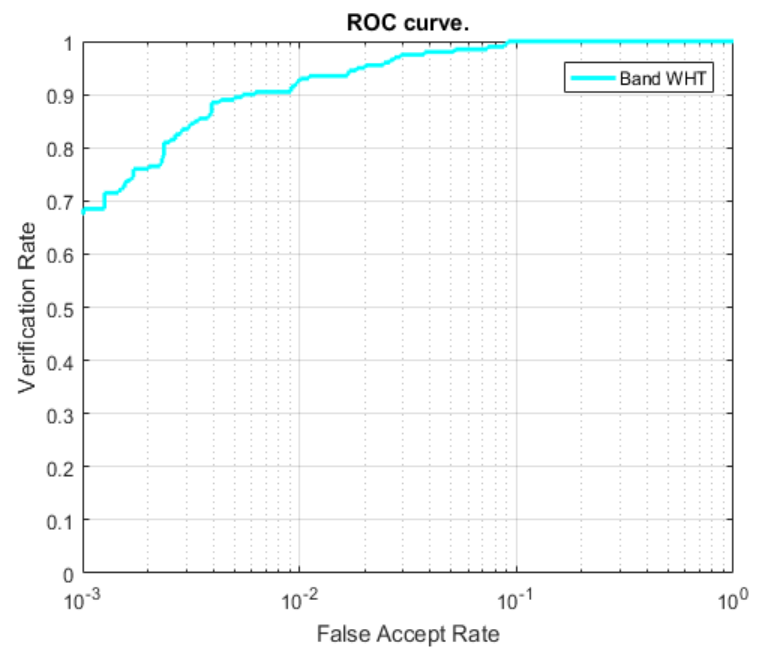

(e)

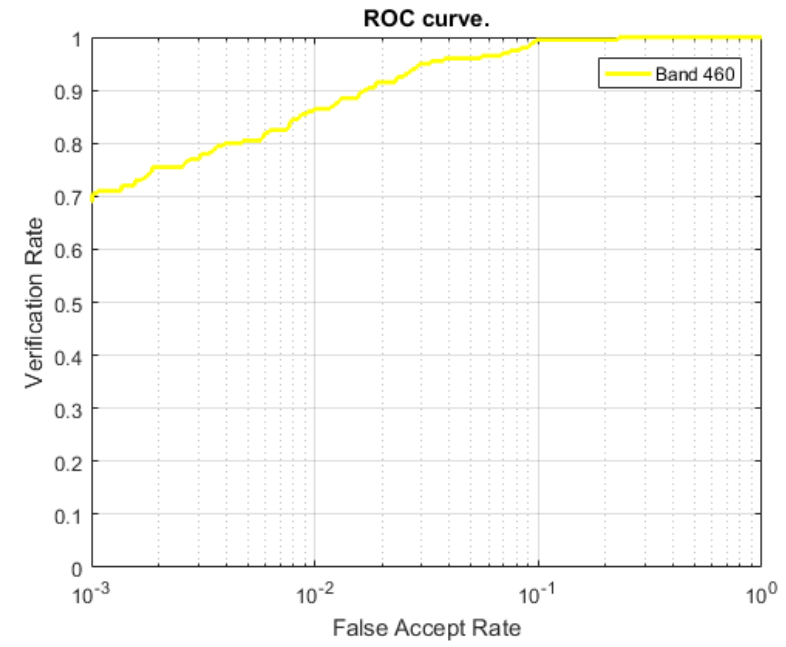

(b)

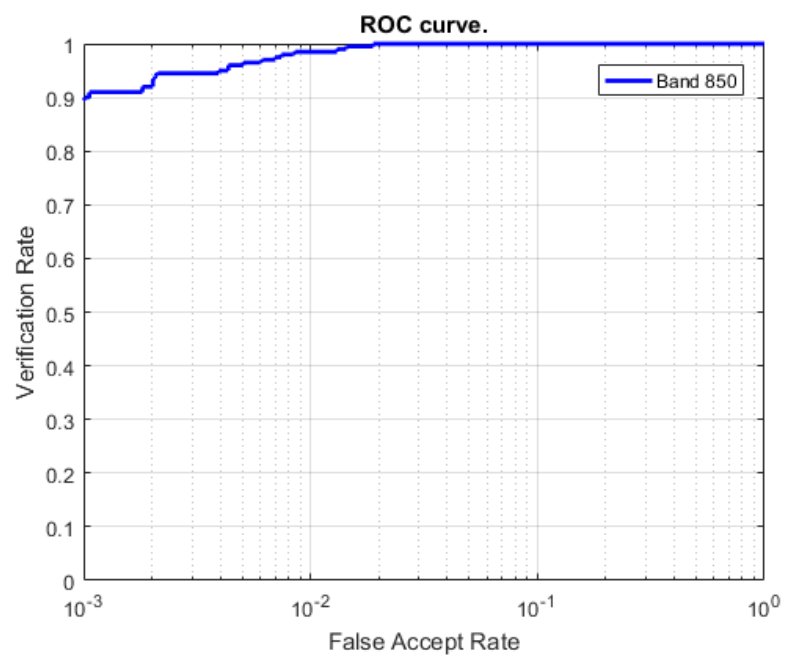

(d)

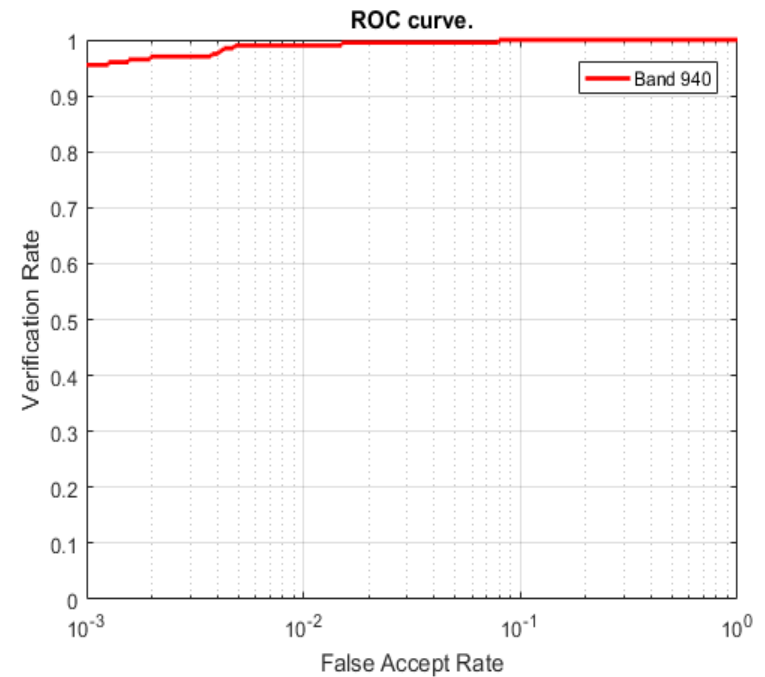

(f)

Fig. 9. ROC Curve for the different Spectral Bands: (a) 630nm Band, (b) 460nm Band, (c) 700nm Band, (d) 850nm Band, (e) WHT Band, (f) 940nm Band. 


\section{CONCLUSION}

In this article, a new palmprint biometric system based on the fusion of multispectral palm images at left hand and right hand level was proposed. This approach is performed by the 2D (DWT) technique during the preprocessing phase to keep more information. Multi-spectral palm images are fused at low level using wavelet transform and decomposition. We used in the main line feature extraction step the Log-Gabor filter which corresponds better to natural images than to other filters to highlight textures. We proposed the technique (KPCA) for the step of reduction of dimension to minimize redundancy. This method is capable of adapting effectively to the variation of rotation, to the potential deformation of the image and to the translation variation. We use the multispectral CASIA database, in which the images are captured with a contactless sensor. Evaluating the performance of our biometric system by applying a Euclidean distance between the vectors from KPCA was considered as the last step. We obtained an ACC rate of 99.50\% for the spectral bands WHT (white light) and $940 \mathrm{~nm}$. Also, the ACC is equal to $99.00 \%$ for the spectral bands 630 $\mathrm{nm}, 850 \mathrm{~nm}, 460 \mathrm{~nm}$ and $98.50 \%$ for the band 700nm. The EER rate achieved by our method is $0.05 \%$ for the bands $(940 \mathrm{~nm}$, WHT) and $1.00 \%$ for the other spectral bands. These results show that our system is robust against spoofing. Our future work will focus on new classification techniques that are more effective in dealing with shadow and unwanted noise. Deep learning architectures like convolutional neural networks (CNNs) need to be studied.

\section{REFERENCES}

[1] X. Yan , W. Kang,"Palm vein recognition based on multi-sampling and feature-level fusion,” Neurocomputing, March (2015),P.798-807 Vol. 151, Part 2, 5.

[2] Z.Khan,A.Mian,Y.Hu, "Contour code: robust and efficient multispectral palmprint encoding for human recognition",in:IEEE International Conference on ComputerVision,(2011),pp.1935-1942.

[3] D.Hong,W.Liu,J.Su,Z.Pan,and G.Wang, "A novel hierarchical approach for multispectral palmprint recognition”, in Neurocomputing,vol 151,part1,(2015),pp.511-521.

[4] A.K.Jain P.Flynn, and A.A.Ross (Eds.), Handbook of biometrics, Springer, USA (2008).

[5] S.J.Daugmanand, The importance of being random:statistic principales of iris recognition ,Pattern Recognit.36(2),(2003),pp. 279-291.

[6] D. Zhang,L. Zhang , and G.Zhenhua , "Feature Band Selection for Online Multispectral Palmprint Recognition," in International Publishing Multispectarl Biometric Springer, (2015), pp.153-162.

[7] W.Song,T.Kim, H.C.kim,J.H.Choi, H.-j.Kong,S.-R.Lee, “Afinger-vein verfication system using mean curvature," in Pattern Recognition Letters 32 ,(2011),pp.1541-1547.

[8] X .Guo, W. Zhou and Y.Zhang,Collaborative representation with hm-lbp features for palmprint recognition. Mach Vis Appl 28(3-4): (2017),pp. 283-291

[9] M. Kusban and A, Budiman, "An excellent system in palmprint recognition," in International Conference on Engineering and Applied Technology (ICEAT),( 2018),pp.1088-1757.

[10] J. Lu,K.N.Plataniotis A.N.Venetsanopoulos , "Face recognition using kernel direct discriminant an alysis algorithms",in IEEE Trans.Neural Netw.14 (1), (2003),pp.117-126.

[11] D.Hong,W.Liu,J.Su,Z.Pan,and G.Wang, "A novel hierarchical approach for multispectral palmprint recognition”, in Neurocomputing,vol 151,part1,(2015),pp.511-521.

[12] R. Raghavendra, B. Dorizzi, A. Rao and G.H. Kumar, "Designing efficient fusion schemes for multimodal biometric system using face and palmprint”, in Pattern Recognit.44(5) (2011),pp.1076-1088.
[13] M.Christopher Bishop, M. Nasser,and Nasrabadi,Pattern recognition and machine learning. Vol. 1, Springer,( 2006), New York.

[14] J.Cui, "2D and 3D Palmprint fusion and recognition using PCA plus TPTSR method".in Neural Comput.Appl. 24, (2014),pp.497-502.

[15] D.Cheng, X.Zhang," “An Improved Recognition Approach for Noisy Multispectral Palmprint by Robust L2 Sparse Representation with a Tensor-Based Extreme Learning Machine,” in Sensors (2019), 19, 235.

[16] R. Kabaciński and M. Kowalski. " Vein pattern database and benchmark results”.In Electronics Letters, (2011),ISSN:pp.1127-1128, vol 47.

[17] A.Al-juboori,1,2 W.Bu, Xi. Wu, and Q.Zhao, "Palm Vein Verification Using Multiple Features and Locality Preaerving Projections", in The Scientific Word Journal Issues, February (2013), Article ID 24603.

[18] A. Al-juboori and W. Bu, "Palm Vein Verification Using Gabor Filter", in ,IJCSI International Journal of Computer Science Issues, January (2013),Vol. 10, Issue 1, No 1.

[19] P.Dubey, T.Kanumuri, "Palmprint Recognition Using Oriented Structural Energy Signature Codes", in Arabian Journal for Science and Engineering. (2019).

[20] M. Tabejamaat, A. Mousavi, Concavity-orientation coding for palmprint recognition, Multimed. Tools Appl., 76 (2017) 9387-940.

[21] L. Fei, G. Lu, Feature extraction methods for palmprint recognition: a survey and evaluation", in IEEE Trans Syst Man Cybern Syst:1-18, (2018).

[22] L. Fei, B. Zhang, Y.Xu, and L Yan, "Palmprint recognition using neighboring direction indicator”.in IEEE Trans. Hum. Mach. Syst.46(6), (2016),pp.787-798.

[23] N. Rose, "Facial expression classification using Gabor and Log-Gabor filters", in: 7th International Conference on Automatic Face and Gesture Recognition, 2006. FGR 2006, (2006), pp. 346-350.

[24] Ross A. Abraham, Karthik Nandakumar, "Anil K. Jain, Handbook of multi-biometrics”, (2006), vol. 6, Springer, USA.

[25] C. Varon, C. Alzate, and J. A. K. Suykens, "Noise Level Estimation for Model Selection in Kernel PCA Denoising", in IEEE Trans. Neural Networks Learn. Syst., vol. 26, no. 11, (2015), pp. 26502663.

[26] L. Shen, L. Bai,M. Fairhurst, "Gabor wavelets and general discriminant analysis for face identification and verification”, Image Vis. Comput. 25 (2007),pp. 552-563.

[27] Y. Hao, Z. Sun, T. Tan and C. Ren, "Multispectral palm image fusion for accurate contact-free palmprint recognition", in: 15th IEEE International Conference on Image Processing, 2008, ICIP 2008, (Oct. 2008), pp. 281284.

[28] S.Manikanda prab, S.N.Sivanandam, "A Novel Biometric system for Person Recognition Using Palm vein Images”, in International Journal on Computer Science and Engineering (IJCSE) ISSN,(2013),pp.0975-3397 Vol. 5 No.

[29] A. Al-juboori and W. Bu, "Palm Vein Verification Using Gabor Filter", in ,IJCSI International Journal of Computer Science Issues, January (2013),Vol. 10, Issue 1, No 1.

[30] S. C. Soh, M. Z. Ibrahim, "Image Fusion based Multi Resolution and Frequency Partition Discrete Cosine Transform for Palm Vein Recognition".in IEEE 6th International Conference on Industrial Engineering and Applications (ICIEA),( 2019).

[31] J.-G. Wang, W.-Y. Yau, A. Suwandy, E. Sung, "Person recognition by fusing palmprint and palm vein images based on "laplacianpalm" representation”, in Pattern Recognit. 41 (May (5)) (2008),pp.1531-1544.

[32] D. Zhang, Z. Guo, G. Lu, L. Zhang and W. Zuo, “An online system of multispectral palmprint verification”, in IEEE Trans. Instrum. Meas. 59 (February (2)) (2010),pp.480-490.

[33] X. Xingpeng, Z. Guo, C. Song and Y. Li, "Multispectral Palm-print Recognition Using a Quaternion Matrix,” in Sensors,(2012),pp. 46334647.

[34] R .Raghavendra, C. Busch, "Novel image fusion scheme based on dependency measure for robust multispectral palmprint recognition”, in Pattern Recognition, 47(6), (2014),pp.2205-2221.

[35] A.Gumaei, R. Sammouda, "An Effective Palmprint Recognition Approach for Visible and Multispectral Sensor Images," in Sensors, 18(5), (2018),1575. 
[36] M.T. Cover, A.T. Joy, "Elements of Information Theory", John Wiley \& Sons, USA, (2012).

[37] K. Huang, S. Aviyente, "Wavelet feature selection for image classification,”in IEEE Trans. Image Process. 17 (9) (2008),pp.17091720.

[38] X. Zhitao, G. Chengming, Y. Ming, L. Qiang, "Research on Log Gabor wavelet and its application in image edge detection," in: Proceedings of 6th International Conference on Signal Processing (ICSP-2002), USA, (2002), pp. 592-595.

[39] M.Kusban,A.Susanto. "Combination a Skeleton Filter and Reduction Dimension of KernelPCA-Based on Palmprint Recognition", in International Journal of Electrical and Computer Engineering (IJECE)Vol. 6, No. 6, December (2016), pp. 3255 - 326.

[40] L. Zhang, L. Li, A. Yang, Y. Shen and M. Yang, “Towards contactless palmprint recognition: A novel device, a new benchmark, and a collaborative representation based identification approach,” in Pattern Recognition, 69 (1) (2017),pp.199-212.

[41] Y. Luo, L. Zhao, B. Zhang, W. Jia, F. Xue, J. Lu, Y. Zhu and B. Xu, "Local line directional pattern for palmprint recognition," in Pattern Recognition, 50(2016),pp.26-44.

[42] Y. Hao, Z. Sun, T. Tan, C. Ren, "Multispectral palm image fusion for accurate contact-free palmprint recognition,” in: 15th IEEE International Conference on Image Processing, 2008, ICIP 2008, (Oct. 2008), pp. 281284.

[43] H. Li, B. Manjunath and S. Mitra, "Multisensor image fusion using the wavelet transform," in Gr. Models Image Process. 57 (3) (1995),pp235245.

[44] Z. Khan, A. Mian and Y. Hu, "Contour code: robust and efficient multispectral palmprint encoding for human recognition," in: IEEE International Conference on Computer Vision, (2011), pp. 1935-1942.

[45] K. Amolins, Y. Zhang and P. Dare, "Wavelet based image fusion techniques - an introduction review and comparison," in ISPRS J. Photogramm. Remote Sens. 62 (4) (2007),pp. 249-263.

[46] R. Raghavendra, B. Dorizzi, A. Rao and G.H. Kumar, "Particle swarm optimization based fusion of near infrared and visible images for improved face verification,” in Pattern Recognit. 44 (2) (2011),pp.401411.
[47] D. Han, Z. Guo and D. Zhang, "Multispectral palmprint recognition using wavelet-based image fusion," in: International Conference on Asian Conference on Computer Vision,(2008), pp. 2074-2077.

[48] K. Huang and S. Aviyente, "Wavelet feature selection for image classification,” in IEEE Trans. Image Process. 17 (9) (2008),pp.17091720.

[49] O.Richard, Duda, E.H.Peter and G.S.David, Pattern classification, John Wiley \& Sons, USA (2012).

[50] N. Japkowicz and M. Shah, "Evaluating Learning Algorithms: A Classification Perspective,” in Cambridge University Press, New York, NY, USA,(2011).

[51] M. Kusban.A, Budiman,“An excellent system in palmprint recognition,” in International Conference on Engineering and Applied Technology(ICEAT),(2018),pp.1088-1757.

[52] M. Kusban and A. Susanto. Combination a Skeleton Filter and Reduction Dimension of KernelPCA-Based on Palmprint Recognition,” in International Journal of Electrical and Computer Engineering (IJECE),(2016),pp.3255-3261.

[53] C. Varon, C. Alzate, and J. A. K. Suykens, "Noise Level Estimation for Model Selection in Kernel PCA Denoising," in IEEE Trans. Neural Networks Learn. Syst., vol. 26, no. 11, (2015),pp. 2650-2663.

[54] X. Yan,W. Kang, F. Deng and Q.Wu, "Palm vein recognition based on multi-sampling and feature level fusion," in Neurocomputing 151(Part 2), (2015),pp.798-807.

[55] D.R. Kisku, A. Rattani, P.Gupta, J.K. Sing, and C.J. Hwang, "Human Identity Verification Using Multispectral Palmprint Fusion," in Journal of Signal and Information Processing, vol. 3, (2012),pp. 263-273.

[56] A.Meraoumia and F. Kadri,"Improving Biometric Identification Performance Using PCANet Deep Learning and Multispectral Palmprint," in Biometric Security and Privacy - Opportunities \& Challenges in The Big Data Era,(2016),pp.51-69.

[57] D.R. Kisku, P. Gupta, J.K. Sing and C. Hwang, "Multispectral palm image fusion for person authentication using ant colony optimization," in Proceedings of International Workshop on Emerging Techniques and Challenges for Hand-Based Biometrics (ETCHB) (IEEE, New York, (2010)), pp. 1-7. 